\title{
General constraints on cross sections deduced from surrogate reactions
}

W. Younes

August 14, 2003

U.S. Department of Energy

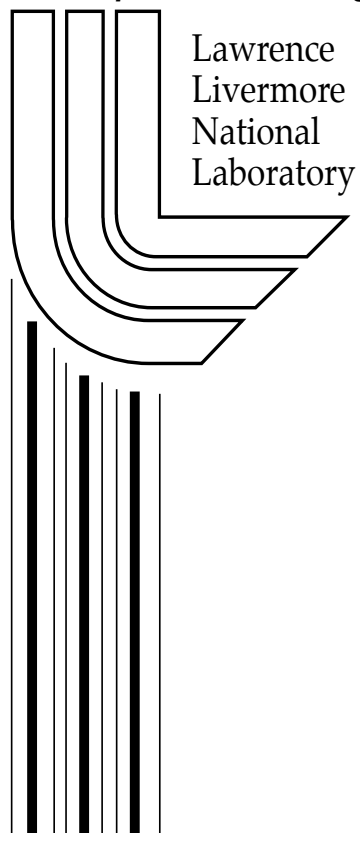


This document was prepared as an account of work sponsored by an agency of the United States Government. Neither the United States Government nor the University of California nor any of their employees, makes any warranty, express or implied, or assumes any legal liability or responsibility for the accuracy, completeness, or usefulness of any information, apparatus, product, or process disclosed, or represents that its use would not infringe privately owned rights. Reference herein to any specific commercial product, process, or service by trade name, trademark, manufacturer, or otherwise, does not necessarily constitute or imply its endorsement, recommendation, or favoring by the United States Government or the University of California. The views and opinions of authors expressed herein do not necessarily state or reflect those of the United States Government or the University of California, and shall not be used for advertising or product endorsement purposes. 


\title{
General constraints on cross sections deduced from surrogate reactions
}

\author{
W. Younes* \\ Lawrence Livermore National Laboratory, Livermore, CA 94551
}

(Dated: August 14, 2003)

\begin{abstract}
Cross sections that cannot be measured in the laboratory, e.g. because the target lifetime is too short, can be inferred indirectly from a different reaction forming the same compound system, but with a more accessible beam/target combination (the "surrogate-reaction" technique). The reactions share the same compound system and a common decay mechanism, but they involve different formation processes. Therefore, an implicit constraint is imposed on the inferred cross section deduced from the measured surrogate-reaction data, through the common decay mechanism. In this paper, the mathematical consequences of this implicit constraint are investigated. General formulas are derived for upper and lower bounds on the inferred cross section, estimated from surrogate data in a procedure which does not require any modeling of the common decay process. As an example, the formulas developed here are applied to the case of the ${ }^{235} \mathrm{U}(n, f)$ cross section, deduced from ${ }^{234} \mathrm{U}(t, p f)$ surrogate data. The calculated bounds are not very tight in this particular case. However, by introducing a few qualitative assumptions about the physics of the fission process, meaningful bounds on the deduced cross section are obtained. Upper and lower limits for the crosssection ratio of the $(n, f)$ reaction on the ${ }^{235} \mathrm{U}$ isomer at $E_{x}=77 \mathrm{eV}$ relative to the $(n, f)$ reaction on the ground state are also calculated. The generalization of this technique to other surrogate reactions is discussed.
\end{abstract}

\section{INTRODUCTION}

In a recent paper [1], neutron-induced fission cross sections on targets of ${ }^{235} \mathrm{U}$ and ${ }^{235} \mathrm{U}^{m}$ have been deduced from fission probabilities measured in the "surrogate" ${ }^{234} \mathrm{U}(t, p f)$ reaction coupled with model calculations of the $n+{ }^{235} \mathrm{U}$ and ${ }^{234} \mathrm{U}(t, p)$ entrance channels. The central idea underlying the surrogate technique in this case is that both $(n, f)$ and $(t, p f)$ reactions can be separated into two sequential and independent steps: (i) a formation process, through the neutron-induced or $(t, p)$ reaction respectively, leading to the same compound system in both cases (i.e. ${ }^{236} \mathrm{U}$ ), and (ii) a decay process, in this case fission, described by the same formalism in both reactions. The separation of the reaction mechanism into two distinct steps is not justified in general if the compound system does not reach an equilibrated state, e.g. in the case of a pre-equilibrium reaction. Fission is a collective phenomenon and therefore implies the existence of an equilibrated system in the decay process. Therefore, fission probabilities measured in the surrogate $(t, p f)$ experiment can be used to constrain the fission model, which is common to both $(n, f)$ and $(t, p f)$ reactions, and the $(n, f)$ cross section can be deduced.

This paper complements the investigation reported in [1] by developing a formalism to impose upper and lower bounds on the cross section deduced from the measured surrogate-reaction data. The limits are based solely on the similarity between formation processes. In other words, there is no need to model the decay process common to both reactions. However, making qualitative assumptions about the decay channel can produce tighter bounds. In the case of the ${ }^{235} \mathrm{U}(n, f)$ cross section deduced from fission probabilities measured in the surrogate ${ }^{234} \mathrm{U}(t, p f)$ reaction, we show that additional qualitative assumptions that capture the gross features of the fission model produce tight bounds on the deduced ${ }^{235} \mathrm{U}(n, f)$ cross section. The results and techniques outlined here can be generalized to other surrogate measurements, regardless of the formation or subsequent decay processes, provided the reaction can be decomposed into these two steps. The ${ }^{235} \mathrm{U}(n, f)$ example will be used to illustrate the concepts. In the extreme case where a surrogate reaction can be selected with formation probabilities (e.g., as a function of spin and parity) very similar to those of the reaction of interest, the formulas derived here can produce meaningful bounds on the inferred cross section, without the need to make any assumptions about the decay mechanism.

\section{CALCULATIONS}

A general expression is derived here that quantifies the extent to which the similarity between the neutroninduced and $(t, p)$ formation processes constrains the $(n, f)$ cross section deduced from measured $(t, p f)$ fission probabilities. Specific reactions are used here for clarity, but the results obtained in this section can be readily generalized. The details of the formalism used in the surrogate technique can be found in [1], and only the necessary equations are repeated here. Fission probabilities, measured in the $(t, p f)$ reaction as a function of the excitation energy $E_{x}$ in the fissioning compound system, can be decomposed as

$$
P_{(t, p f)}\left(E_{x}\right)=\sum_{J^{\pi}} P_{(t, p)}\left(J^{\pi}\right) \times P_{f}\left(E_{x}, J^{\pi}\right)
$$


where the summation extends over the spins and parities $J^{\pi}$ populated by the $(t, p)$ reaction, $P_{(t, p)}\left(J^{\pi}\right)$ is the calculated formation probability, and $P_{f}\left(E_{x}, J^{\pi}\right)$ is the reaction-independent fission probability, determined using a statistical model of the fission process. The corresponding equation for the $(n, f)$ probability below the threshold for the $\left(n, n^{\prime} f\right)$ reaction (i.e., second-chance fission) is

$$
P_{(n, f)}\left(E_{n}\right)=\sum_{J^{\pi}} P_{C N}\left(E_{n}, J^{\pi}\right) \times P_{f}\left(E_{x}, J^{\pi}\right),
$$

where $P_{C N}\left(E_{n}, J^{\pi}\right)$ is the compound-nucleus formation probability for the neutron-induced reaction, as a function of $J^{\pi}$ and the neutron energy $E_{n}=E_{x}-B_{n}$, and where $B_{n}$ is the neutron binding energy. The $(n, f)$ cross section $\left(\sigma_{(n, f)}\right)$ is then obtained by multiplying the probability $P_{(n, f)}\left(E_{n}\right)$ in Eq. (2) by the calculated neutroninduced formation cross section $\sigma_{C N}\left(E_{n}\right)[4]$

$$
\sigma_{(n, f)}\left(E_{n}\right)=\sigma_{C N}\left(E_{n}\right) \times P_{(n, f)}\left(E_{n}\right) .
$$

The formation probabilities for the $(t, p)$ and neutroninduced reactions, and the fission probabilities must be modeled in Eqs. (1) and (2). The surrogate-reaction data provide significant constraints on the fission model. The probability $P_{(t, p f)}\left(E_{x}\right)$ in the left-hand side of Eq. (1) is measured in the surrogate-reaction experiment, and the parameters of the fission model are adjusted to produce the optimal set of fission probabilities, $P_{f}\left(E_{x}, J^{\pi}\right)$, that are independent of the formation process [5].

The same fission probabilities $P_{f}\left(E_{x}, J^{\pi}\right)$ are shared by Eqs. (1) and (2). Therefore, if the formation probabilities $P_{(t, p)}\left(J^{\pi}\right)$ and $P_{C N}\left(E_{n}, J^{\pi}\right)$ are specified and the probability $P_{(t, p f)}\left(E_{x}\right)$ in Eq. (1) is measured, the probability $P_{(n, f)}\left(E_{n}\right)$ in Eq. (2) and the corresponding cross section $\sigma_{(n, f)}\left(E_{n}\right)$ are restricted to a limited range of values, even if explicit $P_{f}\left(E_{x}, J^{\pi}\right)$ values are not specified. In the extreme case where the $(t, p)$ and neutron-induced reactions populate the same states in the compound system, i.e. when $P_{(t, p)}\left(J^{\pi}\right)=P_{C N}\left(E_{n}, J^{\pi}\right)$ for all $J^{\pi}$ at a given $E_{n}$, Eqs. (1) and (2) imply that $P_{(n, f)}\left(E_{n}\right)=$ $P_{(t, p f)}\left(E_{x}\right)$, regardless of the values of $P_{f}\left(E_{x}, J^{\pi}\right)$ calculated using a fission model. In practice, the $(t, p)$ and neutron-induced reaction processes do not produce identical fissioning systems, and we will now derive explicit formulas that quantify the bounds on the deduced cross section, based entirely on the similarity between the formation processes. Although we are using the ${ }^{235} \mathrm{U}(n, f)$ cross section as an example, the results derived here are more general. Therefore, we adopt the following notation to conceal the explicit dependence on the reaction mechanism and specific quantum numbers involved,

$$
\begin{gathered}
p_{i} \equiv P_{C N}\left(E_{n}, J^{\pi}\right), \\
q_{i} \equiv P_{(t, p)}\left(J^{\pi}\right),
\end{gathered}
$$

$$
\begin{gathered}
\epsilon_{i} \equiv P_{f}\left(E_{x}, J^{\pi}\right) \\
P \equiv P_{(n, f)}\left(E_{n}\right)=\sum_{i=1}^{N} p_{i} \epsilon_{i} \\
Q \equiv P_{(t, p f)}\left(E_{x}\right)=\sum_{i=1}^{N} q_{i} \epsilon_{i}
\end{gathered}
$$

defined at a given energy $E_{n}$ (or, equivalently, at excitation energy $E_{x}$ in the compound system), and where the index $i$ stands for the spins and parities $J^{\pi}$ of $N$ states populated in the compound system. In mathematical terms, the problem then is to determine the set of values $\left\{\epsilon_{i}\right\}$ that maximizes (minimizes) the quantity $P$, given a set of values for $\left\{p_{i}\right\},\left\{q_{i}\right\}$, and $Q$, and subject to the constraints

$$
\begin{gathered}
\sum_{i=1}^{N} q_{i} \epsilon_{i}=Q \\
0 \leq \epsilon_{i} \leq 1 \\
0 \leq p_{i} \leq 1 \text { and } \sum_{i=1}^{N} p_{i}=1 \\
0 \leq q_{i} \leq 1 \text { and } \sum_{i=1}^{N} q_{i}=1
\end{gathered}
$$

This is a standard problem in linear programming, solved numerically by the "simplex algorithm" [2]. Eq. (5) defines a convex polyhedron or "simplex" in the coordinate space of the $\epsilon_{i}$ variables, and it can be shown that the maximizing (minimizing) solution for any linear function $P$ of the $\epsilon_{i}$, is always one of the vertices of the simplex. The simplex algorithm is an efficient way of finding the solution vertex numerically. Instead of resorting immediately to the numerical algorithm, we will first derive the desired upper and lower limit on $P$ in a more useful, explicit form by applying the constraints in Eq. 5.

The derivation requires that a distinction be made between those states that are populated by the surrogate reaction (i.e., for which $q_{i} \neq 0$ ) and those that are not (i.e., for which $q_{i}=0$ ). For example, the $(t, p)$ reaction only populates natural-parity states $\left(e . g ., 0^{+}, 1^{-}\right.$, $2^{+}$, etc.) under the assumption that the transferred neutron pair is in a state with relative angular momentum $\ell=0$. For clarity, the index $i$ will refer to states for which $q_{i} \neq 0$, and the primed index $i^{\prime}$ will denote states for which $q_{i^{\prime}}=0$. Let $n$ be the number of states with 
$q_{i} \neq 0$ (i.e., $\left.i=1, \cdots, n\right)$, and $m$ the number of states with $q_{i^{\prime}} \neq 0$ (i.e., $i^{\prime}=1, \cdots, m$ ), so that $m+n=N$. For those $n$ states with $q_{i} \neq 0$, the one-to-one mapping between the indices $i$ and the spin and parity $J^{\pi}$ can be chosen freely. In particular, if that mapping is chosen such that the ratios $p_{i} / q_{i}$ are ordered in a descending sequence (i.e., $p_{1} / q_{1} \geq p_{2} / q_{2} \geq \cdots p_{n} / q_{n}$ ), it can be shown (see Appendix A) that $P$ is bounded by

$$
\sum_{i=k+1}^{n} p_{i}+\frac{p_{k}}{q_{k}}\left(Q-\sum_{i=k+1}^{n} q_{i}\right) \leq P \leq \sum_{i=1}^{j-1} p_{i}+\frac{p_{j}}{q_{j}}\left(Q-\sum_{i=1}^{j-1} q_{i}\right)+\sum_{i^{\prime}=1}^{m} p_{i^{\prime}}
$$

where $j$ is largest index for which $\sum_{i=1}^{j-1} q_{i} \leq Q$, and $k$ is the smallest index for which $\sum_{i=k+1}^{n} q_{i} \leq \bar{Q}$.

In the case of the $(n, f)$ cross section, for example, Eq. (6) gives upper and lower bounds on the $(n, f)$ reaction probability, $P_{(n, f)}$, which is related to the cross section by Eq. (3). The bounds in Eq. (6) depend only on the formation probabilities $\left(p_{i}\right.$ and $\left.q_{i}\right)$, and the measured reaction probability for the surrogate $(t, p f)$ reaction $(Q)$, but not on the fission probabilities $\epsilon_{i}$.

\section{DISCUSSION}

In the limit where the formation processes for the reaction of interest and the surrogate reaction become identical, $p_{i}=q_{i}$ and the sum over $i^{\prime}$ in Eq. (6) disappears. In this special case, the upper and lower limits in Eq. (6) clearly converge to the same value, $Q$, as expected. In the case of the ${ }^{235} \mathrm{U}(n, f)$ cross section, simulated from measured ${ }^{234} \mathrm{U}(t, p f)$ data, the formation processes are markedly different, and therefore Eq. (6) does not provide a tight constraint, as shown in Fig. 1.

The bounds in Eq. (6) can be further constrained if a few, qualitative assumptions are made about the fission probabilities $P_{f}\left(E_{x}, J^{\pi}\right)$ in Eqs. (1) and (2). Stated another way, we will isolate those features of the fission model that are sufficient to reproduce the general behavior of the deduced cross section. In [1], a fairly sophisticated fission model based on the Bohr transition-state hypothesis [3] was used to calculate the $P_{f}\left(E_{x}, J^{\pi}\right)$ values. The hypothesis that low-energy nuclear fission proceeds through states with well-defined quantum numbers was incorporated into a double-humped fission-barrier model. Discrete and continuum states were used in the first well, and on top of each barrier in the model. In this paper, the calculated fission probabilities, $P_{f}\left(E_{x}, J^{\pi}\right)$, are analyzed to reveal their pertinent features. These probabilities are reproduced from [1] in Fig. 2 as a function of excitation energy in the compound system. The $P_{f}\left(E_{x}, J^{\pi}\right)$ curves can be partitioned into three groups. The probabilities of fission from $J^{\pi}=0^{-}$and $1^{+}$compound states are the smallest. As was explained in [1], this is due to the fact that no $0^{-}$states, and only two $1^{+}$states can be constructed below the pairing gaps at the fission saddles. By contrast, the $J^{\pi}=0^{+}, 2^{+}, 4^{+}, 6^{+}$, and $8^{+}$members of

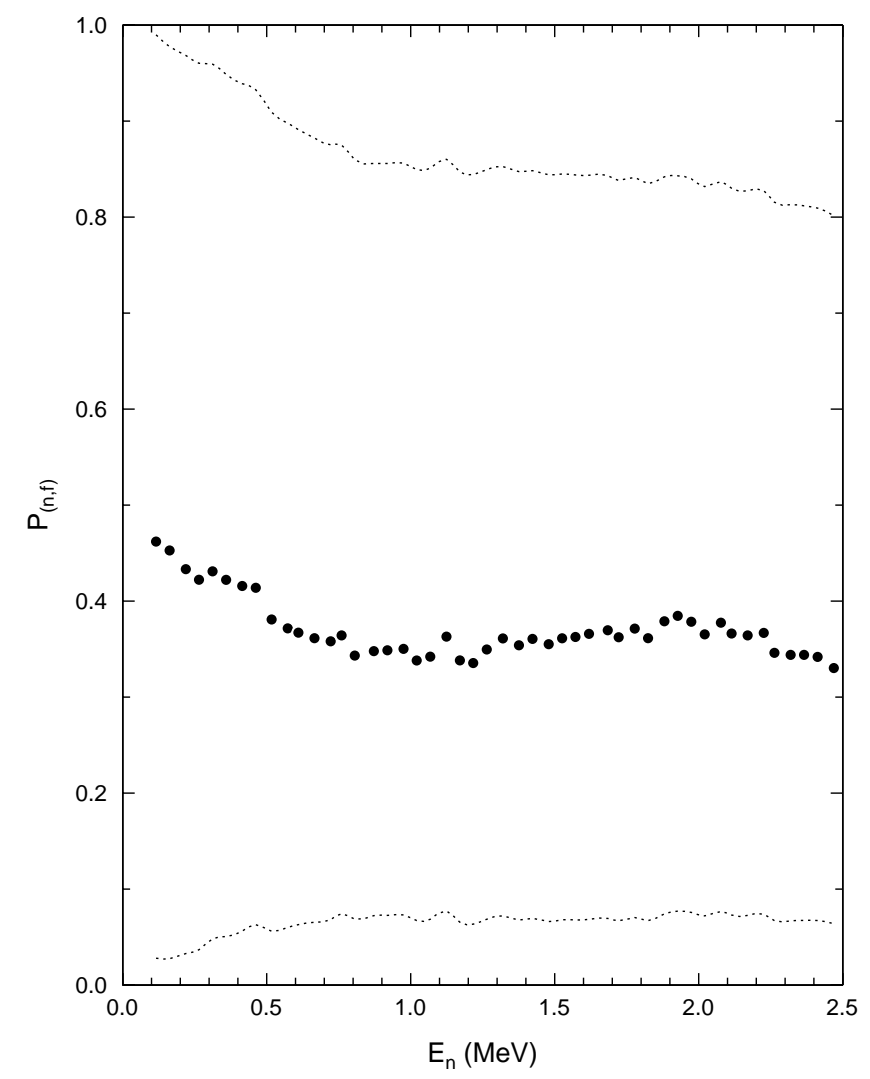

FIG. 1: Fission probability for the ${ }^{235} \mathrm{U}(n, f)$ reaction, plotted as a function of incident neutron energy. The dotted lines show the bounds imposed by Eq. (6), and the filled circles are the exact calculations performed using the model in [1].

the lowest $K=0^{+}$band provide open paths to the fission process starting at the lowest incident neutron energies. The corresponding values of $P_{f}\left(E_{x}, J^{\pi}\right)$ are large. The remaining group of fission probabilities lies somewhere between these extremes.

In the spirit of Eq. (6), we seek to constrain $P_{(n, f)}\left(E_{n}\right)$ assuming that the $P_{f}\left(E_{x}, J^{\pi}\right)$ can be grouped as in Fig. 2, but without any further assumptions about the underlying physics. Using the notation introduced above, we write $P_{f}\left(E_{x}, J^{\pi}\right) \equiv \epsilon_{1}$ for the members of the first $K=$ $0^{+}$band at the saddle, $P_{f}\left(E_{x}, 0^{-}\right)=P_{f}\left(E_{x}, 1^{+}\right) \equiv \epsilon_{3}$, 


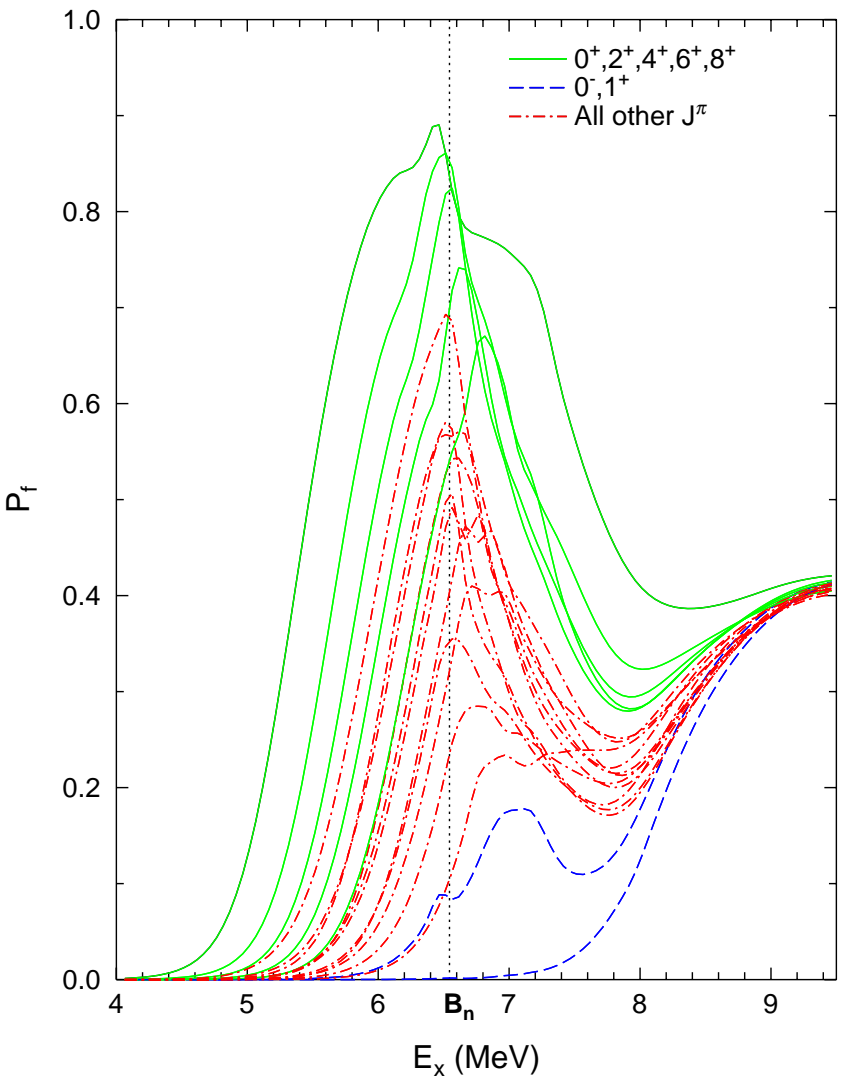

FIG. 2: Fission probabilities $P_{f}\left(E_{x}, J^{\pi}\right)$, calculated in [1] and plotted for different spins and parities as a function of excitation energy. The curves have been classified into three groups, shown as solid, dashed, and dot-dashed lines, and labeled by the appropriate spins in the legend. In particular, the lowest probability curves correspond to fission from the $0^{-}$and $1^{+}$ states. The vertical dotted line marks the neutron binding energy for ${ }^{236} \mathrm{U}, B_{n}$.

and $P_{f}\left(E_{x}, J^{\pi}\right) \equiv \epsilon_{2}$ for all other $J^{\pi}$. Thus, the problem at hand becomes that of extremizing $P=\sum_{i} p_{i} \epsilon_{i}$, subject to the constraints in Eq. (5), and with the additional condition

$$
0 \leq \epsilon_{3} \leq \epsilon_{2} \leq \epsilon_{1} \leq 1
$$

and where only three values of $\epsilon_{i}$, namely $\epsilon_{1}, \epsilon_{2}$, and $\epsilon_{3}$ are allowed. Were it not for the group ordering imposed by constraints in Eq. (7), the solution would be given simply by Eq. (6). Instead, the simplex algorithm is used to provide a numerical solution. The upper and lower limits for the ${ }^{235} \mathrm{U}(n, f)$ probability are plotted in Fig. 3 against the exact result obtained using the model in [1]. Clearly, the additional constraints imposed by grouping the fission probabilities as in Fig. 2 produce tight bounds on the $P_{(n, f)}\left(E_{n}\right)$ values, but no details about the fission model beyond the conditions in Eq. (7) were required.

It is interesting to note that the exact calculation in Fig. 3 reaches and follows the upper bound for $E_{n} \gtrsim$

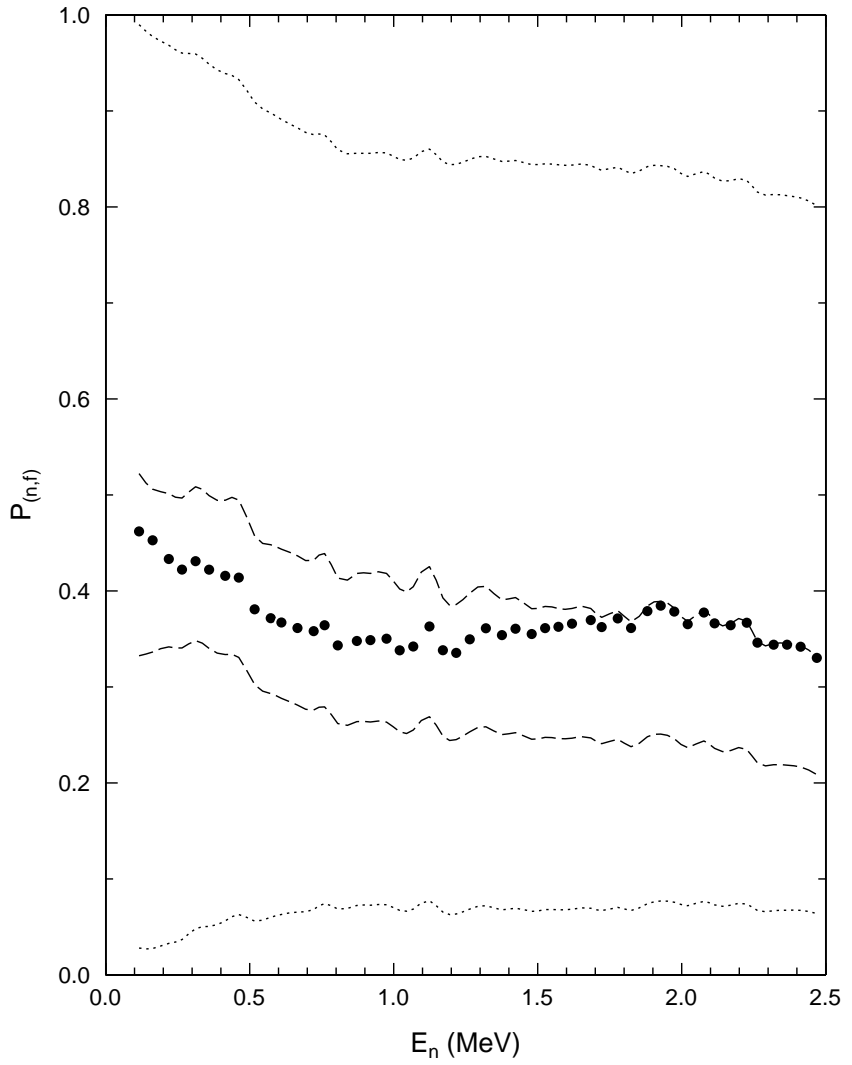

FIG. 3: Bounds (dashed lines) imposed on the ${ }^{235} \mathrm{U}(n, f)$ fission probability by the constraints in Eqs. (5) and (7). The points represent the exact calculation, and are the same as in Fig. 1. The dotted lines are the bounds obtained by using Eq. (6), as in Fig. 1.

1.5 MeV. Specific details of the underlying physics conspire to produce this effect. In the exact calculation [1] for incident neutron energies $E_{n} \gtrsim 1.5 \mathrm{MeV}$, the compound system, ${ }^{236} \mathrm{U}$, is populated at sufficiently-high excitation energies that the open decay channels (i.e., $\gamma$ decay, neutron emission, and fission) are dominated by statistical processes. As discussed in [1], the probabilities $P_{f}\left(E_{x}, J^{\pi}\right)$ become independent of $J^{\pi}$, and tend to the same value required by Eq. (1), namely the measured $(t, p f)$ probability $Q$. Substitution of $P_{f}\left(E_{x}, J^{\pi}\right)=Q$ into Eq. (2) shows that $P_{(n, f)}\left(E_{n}\right)$ also becomes equal to $Q$. On the other hand, the neutron-induced reaction on the $J^{\pi}=7 / 2^{-}$ground state of ${ }^{235} \mathrm{U}$ does not significantly populate the $0^{-}$and $1^{+}$states in the compound system. (The $0^{-}$and $1^{+}$states play a more important role in the neutron-induced fission of the $J^{\pi}=1 / 2^{+}$isomeric level in ${ }^{235} \mathrm{U}$, discussed below.) Furthermore, these unnaturalparity states are not populated at all in the $(t, p)$ reaction. Therefore, the $0^{-}$and $1^{+}$states can safely be ignored in calculating bounds for the ${ }^{235} \mathrm{U}$ ground-state $(n, f)$ probability. The three-group model represented in Fig. 2 reduces effectively to a two-group model. Given the numerical values of the population probabilities for the two remaining groups, it can be shown that the upper limit 
for $P$ is necessarily $Q$. Therefore, both the exact calculation and the upper bound converge to the same value, as is apparent in Fig. 3 .

Finally, the same technique can be applied to derive bounds for the probability of fission in neutron-induced fission of the $\tau \approx 38$-minute isomer at $E_{x}=77 \mathrm{eV}$ in ${ }^{235} \mathrm{U}$. This problem can be approached in the same way as the case of the $(n, f)$ reaction on the ${ }^{235} \mathrm{U}$ ground state discussed above. However, there is a subtle point here that can be used to improve the estimated bounds. Knowledge of the probability for the $(n, f)$ reaction on the ${ }^{235} \mathrm{U}$ ground state provides an additional constraint for the ${ }^{235} \mathrm{U}^{m}$ case, because both share the same set of probabilities $P_{f}\left(E_{x}, J^{\pi}\right)$. Therefore, bounds on the ratio of the fission probability for a ${ }^{235} \mathrm{U}^{m}$ target, relative to the fission probability for a ${ }^{235} \mathrm{U}$ target are calculated. According to Eq. (3), this ratio is identical to the ratio of the corresponding $(n, f)$ cross sections. Extending the notation in Eq. (4), the fission probability for $P_{(n, f)}\left(E_{n}\right)$ for a ${ }^{235} \mathrm{U}^{m}$ target is labeled $P^{(m)}$, and the corresponding formation probabilities are denoted by $p_{i}^{(m)}$. Thus, we wish to calculate bounds for the ratio

$$
\frac{P^{(m)}}{P}=\frac{\sum_{i} p_{i}^{(m)} \epsilon_{i}}{\sum_{i} p_{i} \epsilon_{i}}
$$

subject to the constraints in Eqs. (5) and (7). The simplex algorithm cannot be directly applied because the ratio in Eq. (8) is not a linear function of the variables $\epsilon_{i}$. However, a variation of the method, whereby $P$ is increased in small steps between the bounds in Fig. 3, and the simplex algorithm is used to extremize $P^{(m)}$ at each step is feasible. The extremal values of the ratio obtained from all the incremental steps at each excitation energy are plotted in Fig. 4 against the exact values determined in [1]. At first glance, the bounds do not seem to be very constraining, but it is worth noting that the quantity plotted in Fig. 4 is a ratio of probabilities and can, a priori, assume any value between 0 and $\infty$. In particular, Fig. 4 shows that the probability of fission from the isomer never exceeds the probability of fission from the ground state. This result is a direct consequence of the formation probabilities used and the constraints in Eq. (7), all other details of the fission model are irrelevant.

\section{CONCLUSION}

Upper and lower bounds have been extracted for the neutron-induced fission probability for a ${ }^{235} \mathrm{U}$ target using measured surrogate ${ }^{234} \mathrm{U}(t, p f)$ data. The bounds were deduced without modeling the fission process. Tighter bounds were obtained by further ordering the fission probabilities from states with different spins and parities, based on gross properties of the fission mechanism. The same approach was used to constrain

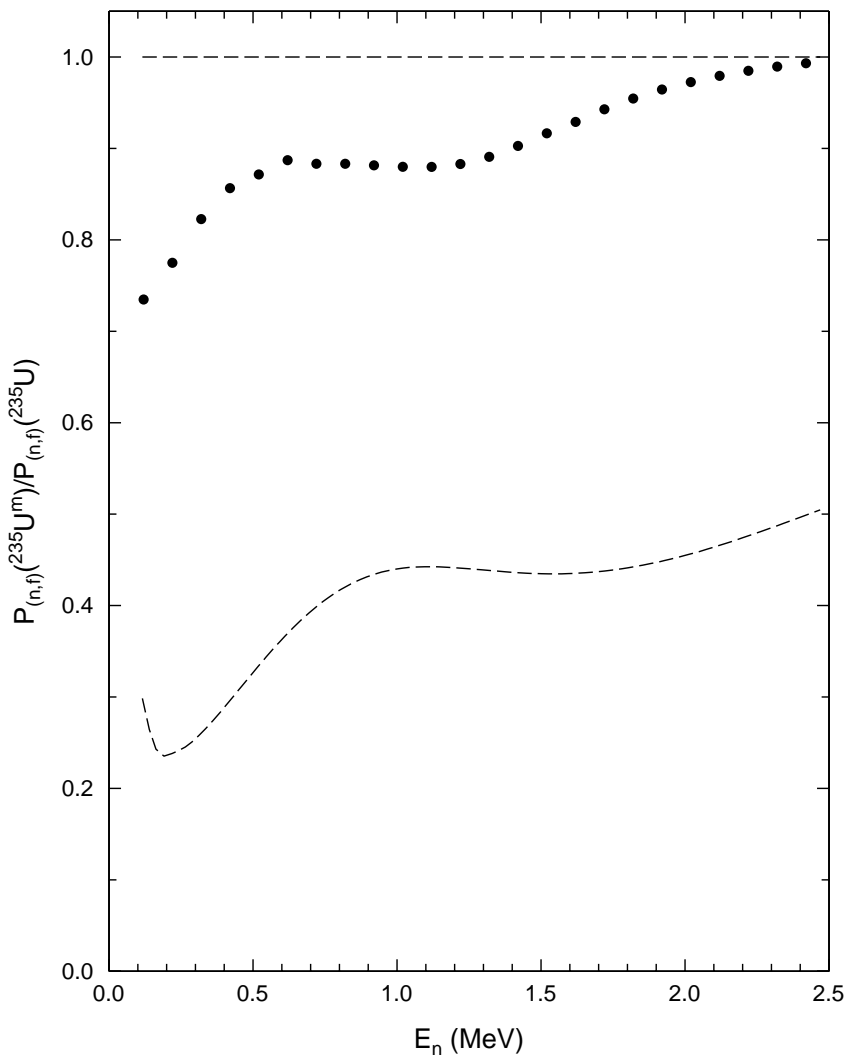

FIG. 4: Bounds (dashed lines) imposed on the isomer-toground-state ratio for ${ }^{235} \mathrm{U}$ by the constraints in Eqs. (5) and (7). This probability ratio is identically equal to the ratio of corresponding cross sections. The points represent the exact calculation [1].

the ratio of the isomer-to-ground-state fission cross sections in ${ }^{235} \mathrm{U}$. The methods discussed above can be applied to a wide range of surrogate-reaction studies. The result in Eq. (6) only requires a description of the formation process, and not of the subsequent decay. In cases where the formation probabilities are more alike than in the $(t, p)$ and neutron-induced reactions discussed here, Eq. (6) may be sufficient to provide tight bounds on the reaction cross section of interest, without the need to develop even a coarse model of the decay process. This may be the case for one-nucleon transfer reactions such as $(d, p),\left({ }^{3} \mathrm{He}, d\right),\left({ }^{3} \mathrm{He}, \alpha\right)$, etc. Otherwise, simple qualitative features of the decay model, such as the ordering of decay probabilities, can be used to produce meaningful constraints.

\section{ACKNOWLEDGMENTS}

I would like to thank J. A. Becker and H. C. Britt for a careful reading of this manuscript and for helpful suggestions. This work was performed under the auspices of the U.S. Department of Energy by the University of California, Lawrence Livermore National Laboratory under 
Contract No. W-7405-Eng-48.

\section{APPENDIX A: PROOF OF EQ. (6)}

The proof is divided into two cases, depending on whether we can assume the all the $q_{i}($ with $i=1, \cdots, N)$ are nonzero or not.

\section{Case 1: $q_{i} \neq 0$}

For convenience, we define

$$
\begin{aligned}
P_{\text {min }} & \equiv \sum_{i=k+1}^{N} p_{i}+\frac{p_{k}}{q_{k}}\left(Q-\sum_{i=k+1}^{N} q_{i}\right) \\
P_{\text {max }} & \equiv \sum_{i=1}^{j-1} p_{i}+\frac{p_{j}}{q_{j}}\left(Q-\sum_{i=1}^{j-1} q_{i}\right),
\end{aligned}
$$

where the indices $i$ are ordered such that $p_{1} / q_{1} \geq p_{2} / q_{2} \geq$ $\cdots p_{n} / q_{n}, j$ is largest index for which $\sum_{i=1}^{j-1} q_{i} \leq Q$, and $k$ is the smallest index for which $\sum_{i=k+1}^{N} q_{i} \leq Q$. It can be seen that $P_{\text {max }}$ is a special value of $P=\sum_{i} p_{i} \epsilon_{i}$ for which the $\epsilon_{i}$ have been chosen as

$$
\begin{aligned}
\epsilon_{1} & =\epsilon_{2}=\cdots=\epsilon_{j-1} \equiv 1 \\
\epsilon_{j} & \equiv \frac{1}{q_{j}}\left(Q-\sum_{i=1}^{j-1} q_{i}\right) \\
\epsilon_{j+1} & =\epsilon_{j+2}=\cdots=\epsilon_{N} \equiv 0 .
\end{aligned}
$$

Direct substitution shows that the $\epsilon_{i}$ in Eq. (A2) satisfy Eq. (5a), as required. These $\epsilon_{i}$ also satisfy Eq. (5b). This is clearly the case for $i=1, \cdots, j-1$ and $i=j+1, \cdots, N$. For $\epsilon_{j}$, we recall that the $j$ was chosen such that it is the largest index for which $\sum_{i=1}^{j-1} q_{i} \leq Q$. Mathematically, this means

$$
\sum_{i=1}^{j-1} q_{i} \leq Q<\sum_{i=1}^{j} q_{i}
$$

Therefore we can write (using the fact that $q_{j} \neq 0$ in the present case)

$$
\begin{aligned}
& Q<\sum_{i=1}^{j} q_{i} \\
\Rightarrow & Q-\sum_{i=1}^{j-1} q_{i}<q_{j} \\
\Rightarrow & \epsilon_{j} \equiv \frac{1}{q_{j}}\left(Q-\sum_{i=1}^{j-1} q_{i}\right)<1 .
\end{aligned}
$$

Similarly, we write

$$
\sum_{i=1}^{j-1} q_{i} \leq Q \Rightarrow \epsilon_{j} \equiv \frac{1}{q_{j}}\left(Q-\sum_{i=1}^{j-1} q_{i}\right) \geq 0,
$$

and therefore $0 \leq \epsilon_{j}<1$, consistent with Eq. (5b).

We are now in a position to show that the set of $\epsilon_{i}$ in Eq. (A2) yields the largest possible value of $P$, i.e. $P \leq P_{\text {max }}$, for any choice of the $\epsilon_{i}$. First we isolate the $\epsilon_{j}$ term in $P=\sum_{i} p_{i} \epsilon_{i}$ and impose the constraint $\sum_{i} q_{i} \epsilon_{i}=Q$ of Eq. (5a) to write

$$
\begin{aligned}
P & =p_{j} \epsilon_{j}+\sum_{i \neq j} p_{i} \epsilon_{i} \\
& =\frac{p_{j}}{q_{j}}\left(Q-\sum_{i \neq j} q_{i} \epsilon_{i}\right)+\sum_{i \neq j} p_{i} \epsilon_{i} \\
& =\frac{p_{j}}{q_{j}} Q+\sum_{i \neq j}\left(\frac{p_{i}}{q_{i}}-\frac{p_{j}}{q_{j}}\right) q_{i} \epsilon_{i} .
\end{aligned}
$$

We rewrite $P_{\max }$ defined in Eq. (A1) in a similar form:

$$
P_{\text {max }}=\frac{p_{j}}{q_{j}} Q+\sum_{i=1}^{j-1}\left(\frac{p_{i}}{q_{i}}-\frac{p_{j}}{q_{j}}\right) q_{i} .
$$

Next, we calculate the difference $P-P_{\max }$

$$
\begin{aligned}
P-P_{\text {max }}= & \sum_{i \neq j}\left(\frac{p_{i}}{q_{i}}-\frac{p_{j}}{q_{j}}\right) q_{i} \epsilon_{i}-\sum_{i=1}^{j-1}\left(\frac{p_{i}}{q_{i}}-\frac{p_{j}}{q_{j}}\right) q_{i} \\
= & \sum_{i=1}^{j-1}\left(\frac{p_{i}}{q_{i}}-\frac{p_{j}}{q_{j}}\right) q_{i}\left(\epsilon_{i}-1\right) \\
& +\sum_{i=j+1}^{N}\left(\frac{p_{i}}{q_{i}}-\frac{p_{j}}{q_{j}}\right) q_{i} \epsilon_{i}
\end{aligned}
$$

Now recall that we have ordered the ratios $p_{i} / q_{i}$ such that $p_{1} / q_{1} \geq p_{2} / q_{2} \geq \cdots \geq p_{N} / q_{N}$, and that $0 \leq \epsilon_{i} \leq 1$, $0 \leq q_{i} \leq 1$ (Eqs. (5b) and (5d), respectively). Therefore, both sums in Eq. (A8) are less than or equal to zero. Thus we have $P-P_{\max } \leq 0$, or $P \leq P_{\max }$.

To prove that $P \geq P_{\text {min }}$, we note that $P_{\text {min }}$ is a special case of $P=\sum_{i} p_{i} \epsilon_{i}$ with the following values for the $\epsilon_{i}$

$$
\begin{aligned}
\epsilon_{1} & =\epsilon_{2}=\cdots=\epsilon_{j-1} \equiv 0 \\
\epsilon_{j} & \equiv \frac{1}{q_{k}}\left(Q-\sum_{i=k+1}^{N} q_{i}\right) \\
\epsilon_{j+1} & =\epsilon_{j+2}=\cdots=\epsilon_{N} \equiv 1 .
\end{aligned}
$$

where $k$ is the smallest index for which $\sum_{i=k+1}^{n} q_{i} \leq Q$. A proof similar to the one for $P \leq P_{\max }$ follows. 
2. Case 2: $q_{i} \neq 0, q_{i^{\prime}}=0$

In this case, the values of $\epsilon_{i^{\prime}}$ do not affect the sum $\sum_{i} q_{i} \epsilon_{i}+\sum_{i^{\prime}} q_{i^{\prime}} \epsilon_{i^{\prime}}=Q$ in Eq. (5a). Therefore, we are free to choose any values of $0 \leq \epsilon_{i^{\prime}} \leq 1$, and still satisfy the constraint in Eq. (5a). In particular, to maximize $P$, we choose $\epsilon_{i^{\prime}}=1$, and to minimize it we choose $\epsilon_{i^{\prime}}=0$. Thus we have the general result

$$
\begin{aligned}
P_{\text {min }} & \equiv \sum_{i=k+1}^{n} p_{i}+\frac{p_{k}}{q_{k}}\left(Q-\sum_{i=k+1}^{n} q_{i}\right) \\
P_{\text {max }} & \equiv \sum_{i=1}^{j-1} p_{i}+\frac{p_{j}}{q_{j}}\left(Q-\sum_{i=1}^{j-1} q_{i}\right)+\sum_{i^{\prime}=1}^{m} p_{i^{\prime}},(\mathrm{A} 10)
\end{aligned}
$$

[1] W. Younes and H. C. Britt, Phys. Rev. C 67, 024610 (2003).

[2] W. H. Press, B. P. Flannery, S. A. Teukolsky, and W. T. Vetterling, Numerical Recipes in C: The Art of Scientific Computing (Cambridge University Press, 1993), 2nd ed.

[3] A. Bohr, in Proceedings of the First United Nations International Conference on the Peaceful Uses of Atomic Energy (1955), vol. 2, p. 151.

[4] In reference [1], Eqs. (2) and (3) are combined into a single where the summations over $i$ now cover the indices for which $q_{i} \neq 0$. equation. The equations are kept separate here so that the discussion may focus on probabilities, rather than cross sections.

[5] In practice, the measured $P_{(t, p f)}\left(E_{x}\right)$ values could not be exactly reproduced, due to limitations in the fission model. A renormalization procedure, described in [1] has been used to compensate for these limitations. 